\title{
Low-energy antikaon nucleon interaction physics revisited
}

\author{
Johann Marton ** \\ Stefan Meyer Institute for Subatomic Physics, Austrian Academy of Sciences. \\ E-mail: iohann.marton@oeaw.ac.at
}

\begin{abstract}
The strong interaction of antikaons with nucleons and nuclei at low energies is subject to intense studies in theory and experiment. The topics under study cover precision experiments with kaonic atoms, the study of the nature of the famous sub-threshold $\Lambda(1405)$ resonance and the search of lively discussed antikaon induced nuclear bound states. This talk will give an overview of the different research topics included in the LEANNIS network of the EU integrated activity HadronPhysics2. The present status and perspectives in this scientific field are discussed.
\end{abstract}

XLIX International Winter Meeting on Nuclear Physics, BORMIO2011

January 24-28, 2011

Bormio, Italy

\footnotetext{
* Speaker.

${ }^{\dagger}$ Work partly supported by EU within I3-HadronPhysics2 Contract No. 227431
} 


\section{Introduction}

The strong interaction with strangeness at low energies is a challenging field for experiments and theory. Exotic atoms with hadrons substituting the electron (so called hadronic atoms) provide the access to relevant observables. Especially interesting are kaonic atoms where an electron is substituted by a negatively charged kaon $\mathrm{K}^{-}$called antikaon $\bar{K}(\mathrm{~S}=-1)$. Due to the larger mass of the antikaon than the electron mass $\left(\mathrm{m}_{K^{-}} \sim 1000 \mathrm{~m}_{e}\right)$ the atomic size and the transition energies are modified. Kaonic hydrogen $\mathrm{K}^{-} \mathrm{p}$ represents the simplest hadronic atom with strangeness and therefore the touchstone for the understanding of the low-energy antikaon-nucleon $(\bar{K} \mathrm{~N})$ interaction. The $\bar{K} \mathrm{~N}$ interaction leads to an energy displacement $\varepsilon_{1 s}$ and level broadening $\Gamma_{1 s}$ of the $1 \mathrm{~s}$ ground state (the strong interaction effect on the higher $\mathrm{n}$ states including the $2 \mathrm{p}$ state is negligible) which can be resolved by x-ray spectroscopy. First experimental studies of x-ray transitions in kaonic hydrogen started at CERN and Rutherford Laboratory in the late 1970 and early 1980 by Bird [四], Davies [ [ $]$ ] and Izycki [ []]. These old experiment indicated a puzzling disagreement between the results of kaon scattering experiments and kaonic atom experiments about the sign of the strong interaction shift. This puzzle was solved at KEK [田] and verified by the DEAR experiment [[]]. The atom $\mathrm{K}^{-} \mathrm{p}$ is the prototype kaonic system for precision studies of the $\bar{K} \mathrm{~N}$ interaction by $\mathrm{x}$-ray spectroscopy. Challenges in experiment and theory are the enhancement of the precision and the extraction of the scattering lengths.

The limited intensity of low energy antikaon sources and the rather low x-ray yield (e.g. few $\%$ for the $\mathrm{K}_{\alpha}$ transition in $\mathrm{K}^{-} \mathrm{p}$ even in low density gas) impose experimental challenges. Also hadronic background from kaon correlated events has to be taken into account. Recently new data from the x-ray spectroscopy of the atomic transitions to the $1 \mathrm{~s}$ state (Lyman transitions $\mathrm{K}_{n p \rightarrow 1 s}$ ) are available from the SIDDHARTA experiment at DAFNE. This experiment used modern solid state x-ray detectors, i.e. silicon drift detectors (SDDs) [ [G, 团], which represent a key for kaonic hydrogen studies because of the compactness, the high efficiency at the energies of the $\mathrm{K}$ lines, high energy resolution and the timing capability for suppressing the non-kaon correlated (asynchronous) background.

In the contrary to pionic hydrogen where the pion-nucleon interaction can be successfully described in the framework of chiral perturbation theory (ChPT) the strong interaction in kaonic atoms is more complex due to the presence of resonances like the famous $\Lambda^{*}(1405)$ [ [ $]$ ] resonance of still unclear nature which prohibit ChPT application. The theoretical description is based on effective field theories [9] which have already reached a high level of refinement but need as anchor at threshold the input from precision measurements of the strong interaction parameters of kaonic hydrogen, i.e. $\varepsilon_{1 s}$ and $\Gamma_{1 s}$. The predictive power of current theory for the antikaon interaction below threshold is limited and subject to intense studies because of the implications for the existence of kaonic nuclear bound systems.

Equally interesting is the search for kaonic nuclear clusters (KNC) in which the antikaon $\mathrm{K}^{-}$ could provide the binding of nucleons to form systems like $\mathrm{K}^{-} \mathrm{pp}$ as predicted by Akaishi and Yamazaki in 2002 [ए]]. Recently indications for the existence of this type of bound states were 
claimed to be found [ $[\mathbb{W}]$ and theoretical studies are made [[2]]. Nevertheless, independent verification and studies on the characteristics are still necessary. Other informations about KNC are coming from various experiments like FINUDA [123], FOPI [14] and OBELIX [U15] but other interpretations of the findings are under study [ㅁ]. The present results on KNCs is lively discussed by theoreticians.

New dedicated exclusive experiments like AMADEUS [ए7] and E15 [ए8] at J-PARC are in preparation to investigate the KNC case in detail. A collection of recent work can be found in ref.[एप].

The networking activity LEANNIS [ㅁ] within the European project HadronPhysics2 [ [2]] is bringing together theoreticians and experimentalists for joint work in the field of antikaon interaction with nucleons and nuclei.

\section{Kaonic atom studies at $D A \Phi N E$}

The DФFNE positron-electron collider at LNF is a unique source of low energy nearly monoenergetic $\mathrm{K}^{-}(\sim 16 \mathrm{MeV})$ from the decay of $\Phi$ mesons $\left(\Phi \rightarrow K^{-}+K^{+}\right.$, branching ratio $\left.\sim 50 \%\right)$. SIDDHARTA [22] is taking advantage of this kaon source ideally suited for stopping $\mathrm{K}^{-}$in thin gaseous targets to produce kaonic atoms like kaonic hydrogen and kaonic helium atoms for x-ray spectroscopy. In fig. $\square$ the principle of the experimental method is given. The uncorrelated background can be suppressed by orders of magnitude applying a triple coincidence between the kaon pair and the $\mathrm{x}$ ray . The $\mathrm{x}$-ray spectroscopy was performed with an array of 144 SDDs poviding an active area of $144 \mathrm{~cm}^{2}$ around the gas target.

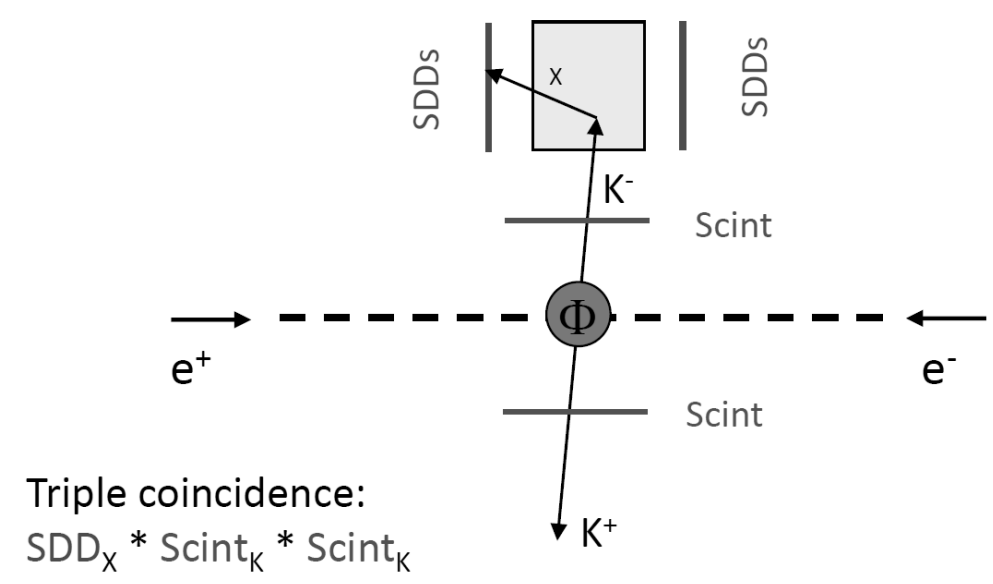

Figure 1: Scheme of the experimental method of SIDDHARTA using a triple coincidence of 2 kaon trigger detectors $\left(\mathrm{Scint}_{K}\right)$ for the $\mathrm{K}^{+}-\mathrm{K}^{-}$pair and the $\mathrm{x}$-ray detectors $\left(\mathrm{SDD}_{X}\right)$. Using this method the asynchronous background can be efficiently suppressed.

\subsection{Kaonic hydrogen}

The most simple systems to study the kaon-nucleon interaction are represented by kaonic hydrogen and kaonic deuterium. The yield of the $\mathrm{K}_{\alpha}$ transition of kaonic hydrogen is in the range of 
1-3\% whereas for kaonic deuterium the $\mathrm{K}_{\alpha}$ yield is anticipated to be smaller by an order of magnitude. Using the experimental method of fig.1 SIDDHARTA succeeded in measuring the Lyman $\mathrm{x}$-ray spectrum of kaonic hydrogen and performed a first study with a pure deuterium gas filling. In fig.2 the arrangement of the SIDDHARTA experiment at the $\mathrm{e}^{+}-\mathrm{e}^{-}$intersection of DAFNE is shown.

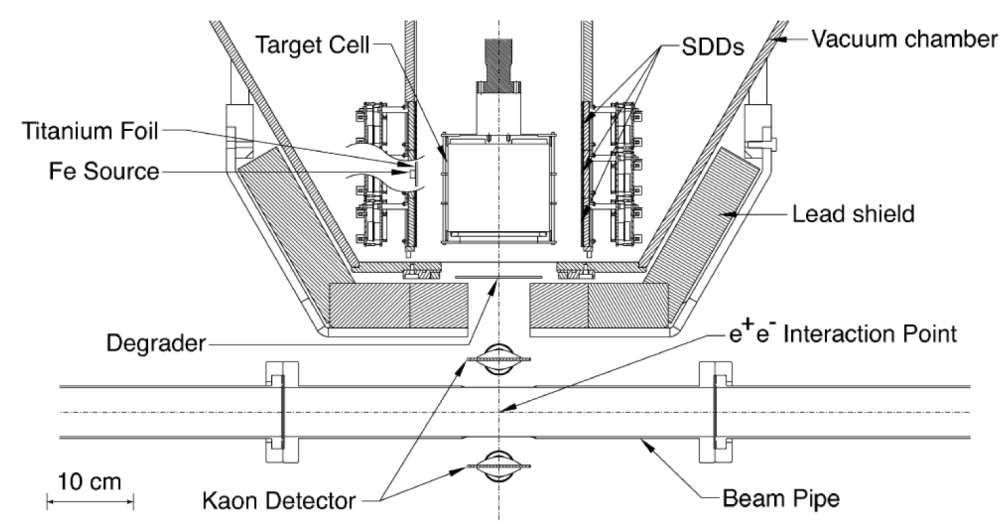

Figure 2: Schematic layout of the SIDDHARTA experiment.

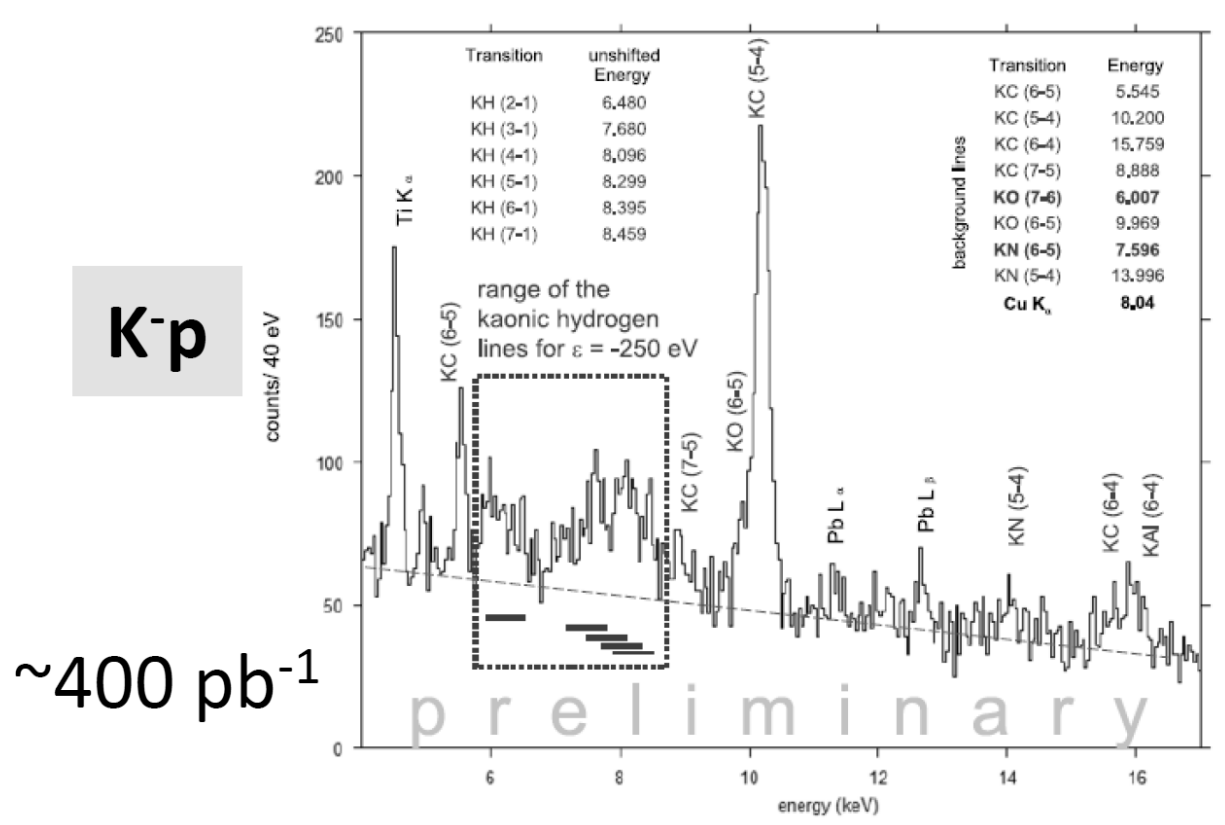

Figure 3: X-ray energy spectrum of kaonic hydrogen measured by SIDDHARTA [23]]. The rectangular frame indicates the energy region of interest with the $\mathrm{K}$-transitions $\left(\mathrm{K}_{\alpha}, \mathrm{K}_{\beta}\right.$ and higher $\mathrm{K}$ lines).

In fig. 4 the preliminary values for $\varepsilon_{1 s}$ and $\Gamma_{1 s}$ of SIDDHARTA are shown which are compatible with the final values which will be published soon [24]. From these values the $\mathrm{K}^{-} \mathrm{p}$ scattering 
Shift and width with $1 \sigma$ error

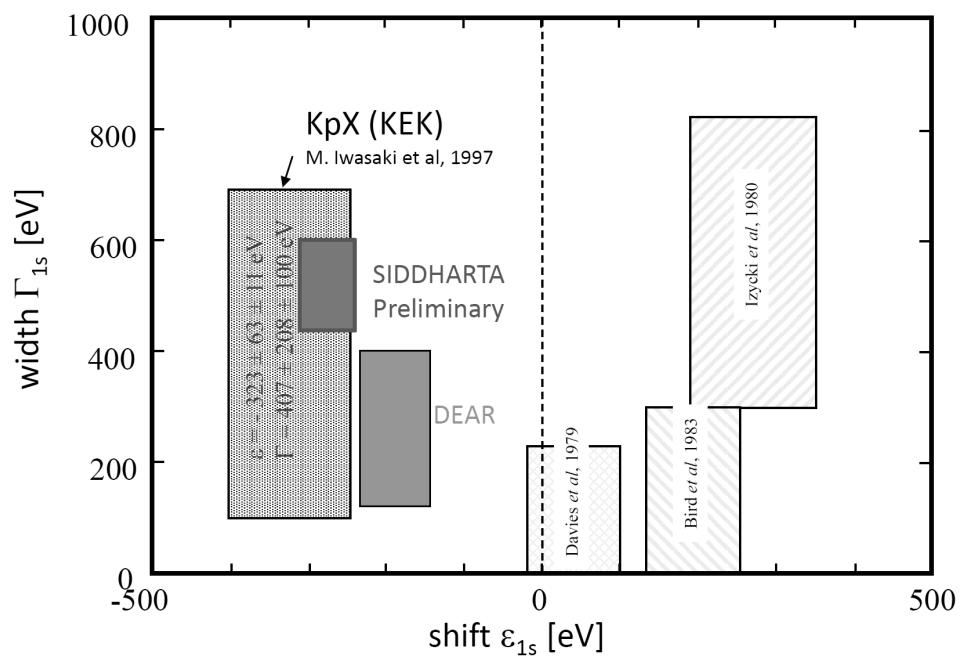

Figure 4: Strong interaction shift versus width measured in different experiments. The newer experiments KpX, DEAR and SIDDHARTA showing a negative value for the shift. Note the remarkable progress in precision by SIDDHARTA.

length can be deduced (see equation below). Here an improved formula[25] extending the traditional Deser-Truman formula by taking isospin breaking into account was developed.

$$
\varepsilon_{1 s}-i \frac{\Gamma_{1 s}}{2}=-2 \alpha^{3} \mu_{c}^{2} a_{p} x\left(1-2 \alpha \mu_{c} a_{p}(\ln \alpha-1)+\ldots .\right)
$$

Her $\mathrm{a}_{p}$ denotes the threshold amplitude, $\mu_{c}$ the reduced mass and $\alpha$ the fine structure constant.

A similar relation exists for the strong interaction observables $\varepsilon_{1 s}$ and $\Gamma_{1 s}$ in kaonic deuterium but also the effect of the nucleon recoil (10-15\% effect) [26] has to be taken into account.

\subsection{Kaonic helium}

Why is kaonic helium such an interesting scientific case? Traditionally the $2 p$ orbit in kaonic helium is assumed to be the last orbit, i.e. the transition to the 1s state would be unobservable. In former experiments a large effect (in the order of $40 \mathrm{eV}$ ) of the strong interaction on the $2 \mathrm{p}$ state was claimed which resulted in a puzzling situation because theory predicted much smaller values for the $2 \mathrm{p}$ state shift $\left(\varepsilon_{2 p} \sim 0 \mathrm{eV}\right)$. In an experiment at KEK a small value of the $2 \mathrm{p}$ state shift was found (see below).

X-ray data on the strong interaction in kaonic helium $\left(\mathrm{K}^{4} \mathrm{He}, \mathrm{K}^{3} \mathrm{He}\right)$ and kaonic hydrogen and deuterium were taken in 2009. The strong interaction shift of the $2 p$ state of $\mathrm{K}^{4} \mathrm{He}$ was extracted from these data [27] confirming the result of the former KEK experiment on $\mathrm{K}^{4} \mathrm{He}$ [28].

$$
\begin{gathered}
\varepsilon_{2 p}\left({ }^{4} \mathrm{He}\right)=0 \pm 6(\text { stat. }) \pm 2(\text { syst. }) e V(\text { SIDDHARTA }, 2009) \\
\varepsilon_{2 p}\left({ }^{4} \mathrm{He}\right)=+2 \pm 2(\text { stat. }) \pm 2(\text { syst. }) e V(\text { KEK }, 2007)
\end{gathered}
$$


The strong interaction on the $2 \mathrm{p}$ state is compatible with 0 thus discarding the results of former experiments which gave a value of $\varepsilon_{2 p}\left({ }^{4} \mathrm{He}\right)$ which could not be explained by theory. SIDDHARTA succeeded in measuring the Balmer x-ray spectrum of kaonic ${ }^{3} \mathrm{He}$ for the first time [2Q].

$$
\varepsilon_{2 p}\left({ }^{3} \mathrm{He}\right)=-2 \pm 2(\text { stat. }) \pm 4(\text { syst. }) e V(\text { SIDDHARTA, 2011) }
$$

The question arises if there is a (small) isotopic difference which has been predicted by Akaishi [B] $]$. Unfortunately this question cannot be answered by our results due to the statistical and systematic errors.

An experiment on kaonic ${ }^{3} \mathrm{He}$ with a liquid target is in preparation at J-PARC which is aiming at an improvement of the precision [3]].

Regarding the $\mathrm{K}$ transitions in kaonic helium there are no data available because of the reduced dynamic range of the newer experiments the energy region of $\mathrm{K}$ transition $2 \mathrm{p}-1 \mathrm{~s}$ (around $30 \mathrm{keV}$ ) could not be studied. In the future attempts will be made in SIDDHARTA2 to search for the K transitions which should be strongly affected by the strong interaction. However, the caveat is the unknown and probably very low $\mathrm{x}$-ray yield.

\section{Perspectives for new experimental studies}

\subsection{Kaonic deuterium}

In order to extract the isospin-dependent $\mathrm{S}$-wave scattering lengths $\mathrm{a}_{0}$ and $\mathrm{a}_{1}$ the strong interaction shift and width of kaonic hydrogen is not sufficient - one needs the same quantities for kaonic deuterium. The kaonic deuterium measurement is significantly more complicated since the yield of the $\mathrm{K}$ lines is lower by an order of magnitude [32, [33] compared with the kaonic hydrogen case. On the other hand the theoretical predictions for the energy shift and width are spanning a wide range (shift from several hundred $\mathrm{eV}$ to nearly $1 \mathrm{keV}$, width from several hundred $\mathrm{eV}$ to several $\mathrm{keV}$ ) [B4, [35]. For the first time the SIDDHARTA experiment made the attempt to measure the the $\mathrm{K}$ line $\mathrm{x}$-ray spectrum. Due to the limited beam time and the obviously low X-ray yield no significant $\mathrm{K}$ lines could be extracted. With much improved experimental techniques new spectroscopic measurements of kaonic deuterium are foreseen in the follow-up experiment SIDDHARTA2 at LNF [B6].

\section{2 $\Lambda(1405)$ and $\Sigma(1385)$ Resonances}

The sub-threshold behavior of the $\bar{K} \mathrm{~N}$ interaction is very much different to the $\pi \mathrm{N}$ interaction and characterized by resonances $\Lambda(1405)$ in the s wave and $\Sigma(1385)$ in the p wave which lead to many surprises.

This characteristics calls for questions which are subject to intense investigations in experiment and theory:

What role is $\Lambda(1405)$ playing and what is its nature?

Has $\Lambda(1405)$ a 2-pole structure and can this structure be studied in experiments in more detail? Originally it was assumed by Yamazaki and Akaishi [ए]] that $\Lambda(1405)$ is a $\mathrm{K}^{-} \mathrm{p}$ bound state acting as a "doorway" to other deeply bound nuclei like $\mathrm{K}^{-} \mathrm{pp}$. This conjecture is still heavily discussed in theory (see below). In this context there are new experimental data on the shape of $\Lambda(1405)$ from 


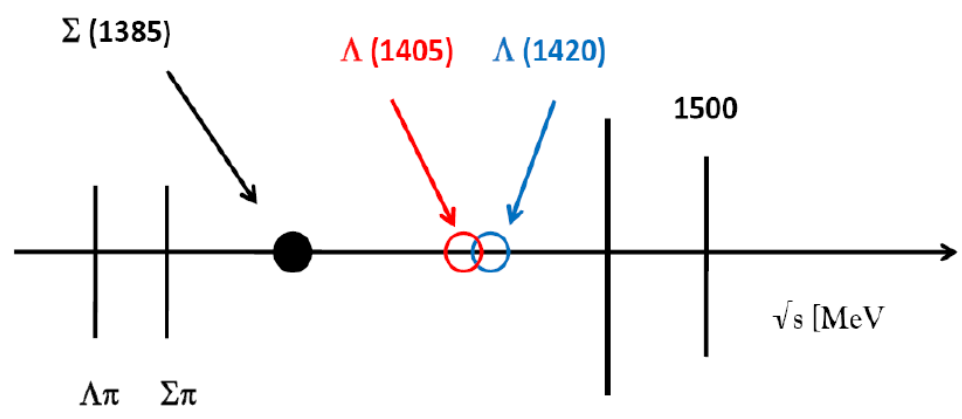

$\overline{\mathbf{K} N}$

Figure 5: Resonances in the sub-threshold domain of the $\bar{K} \mathrm{~N}$ interaction.

HADES[B7] and CLAS [B8] which might halp to clarify the structure. A new method to study $\Lambda$ (1405) was proposed by S. Wycech [39] by detection of the $\gamma$ from the reaction $\mathrm{K}^{-} \mathrm{p} \rightarrow \Lambda+\gamma$ which is included in the experimental program of SIDDHARTA2.

\subsection{Kaonic nuclei}

The $\bar{K}$-N threshold data are an essential input for the studies of kaonic nuclei about which is still a controversy among theoreticians. Experimental indications of kaonic nuclei and theoretical studies do not agree on a consistent picture about their binding energies and decay widths.

- What is the production mechanism of kaonic nuclei (deeply-bound kaonic nuclear clusters)? What are the binding energies and the widths of possibly formed kaonic nuclei?

- What is the density of such kaonic nuclei? Is it possible to produce cold dense matter by producing kaonic nuclei with strangeness $\mathrm{S}=-2$ and exploring the QCD phase diagram at these conditions?

Several experiments employing different methods like DISTO (proton-proton collision)[W], FINUDA ( $\mathrm{K}^{-}$induced reaction)[[3]] and OBELIX (antiproton-induced reaction) [[15]) found indications for the existence of kaonic nuclei. In this context new data will come from experiments at GSI with the FOPI detector using the reaction $p+p \rightarrow K \Lambda p$ [40]].

Concerning theory the results of phenomenological studies with the Ansatz $\Lambda(1405) \doteq \mathrm{K}^{-} \mathrm{p}$ (AY02) [ए]], Faddeev calculations (FC) [4], 42, 43] and variational calculations (VC) [44, 45] on the properties of $\mathrm{K}^{-} \mathrm{pp}$ cover a broad range. Different results were obtained for the binding energy ranging from 45-80 MeV (FC) to 20-80 MeV (VC). Results for the decay width cover 45-110 $\mathrm{MeV}$ (FC) to 40-85 MeV (VC). The comparison experimental values for the binding energy and width with theoretical expectations give a quite incoherent picture. Clearly the experimental and theoretical studies on kaonic nuclei need still improvements.

New fully exclusive experiments like AMADEUS [46] and E15 [47] at J-PARC are in preparation . The goal of AMADEUS is the exclusive study of the production and decay of kaonic nuclei produced by antikaon absorption using a modified KLOE setup at the DA $\Phi$ NE $\Phi$-factory 
in Frascati. The AMADEUS apparatus will provide the detection of all charged, and neutral outgoing particles including photons using the $4 \pi$ detector system of KLOE, cryogenic targets and a kaon tagging system similar to SIDDHARTA. The detector systems will employ new technologies like GEM based detectors [48] and photo-detection with silicon photomultipliers (SiPMs) [49]. The goals will be, besides high statistics studies of various absorption processes of stopped anti-kaons in light nuclei, the possible unambiguous detection of various light anti-kaonic nuclear systems, such as $\mathrm{K}^{-} \mathrm{pp}, \mathrm{K}^{-} \mathrm{ppn}$, and $\mathrm{K}^{-}$pnn with data on the binding energies, total widths and all partial decay widths. From Dalitz plots of 3-body decays of kaonic nuclei detailed structure information (such as parity, angular momentum, radii and densities) can be deduced. Such data would give valuable input in theories for the description of dense, cold, strongly interacting systems. In Japan a new technique (in-flight production of kaonic nuclei) will be used in the E15 experiment at J-PARC. This experiment is in preparation now.

\section{Summary}

The low-energy anti-kaon interaction physics is a research field characterized by some puzzles found in the kaonic hydrogen and kaonic helium cases which were solved in the last years. Nevertheless challenging problems remain and are subject to new experiments and theoretical work. The LEANNIS network within the European project HadronPhysics2 was established to comprise strangeness physics projects which deal with low energy interactions of anti-kaons with nucleons and nuclei. The activities are concentrated on developing new strategies in the experimental and theoretical sectors, to attack the numerous problems in the field. The development of new experimental methods and techniques, e.g. SDDs as high-performance x-ray detectors, GEM based detectors and new SiPM photon detectors will establish a strong instrumental basis for future experiments. The R\&D work on GEM and SiPM based detectors are integrated in the HadronPhysics2 project. Major European institutes working in this field are participating to the LEANNIS network, therefore a platform is given to promote and focus the efforts. A close collaboration of experimentalists with theoreticians, for the analysis of the new upcoming experimental results and their interpretation in the framework of the non-perturbative QCD with strangeness is an essential goal of this network in order to further develop this research field and gain a detailed understanding of the many facets of low-energy anti-kaon nucleon and -nucleus interactions.

\section{Acknowledgements}

Part of this work was supported by Hadron- Physics I3 FP6 European Community program, Contract No. RII3-CT- 2004-506078; the European Community Research Infrastructure Integrating Activity - Study of Strongly Interacting Matter- (HadronPhysics2, Grant Agreement No. 227431) under the Seventh Framework Programme of EU; Austrian Federal Ministry of Science and Research 650962/0001 VI/2/2009.

\section{References}

[1] P. M. Bird et al., Nucl. Phys. A 404, 482 (1983). 
[2] J. D. Davies et al., Phys. Lett. B 83, 55 (1979).

[3] M. Izycki et al., Z. Phys. A 297, 11 (1980).

[4] M. Iwasaki et al. [KpX Collaboration], Phys. Rev. Lett. 78 (1997) 3067; T.M. Ito et al. [KpX Collaboration], Phys. Rev. C58 (1998) 2366.

[5] G. Beer et al. [DEAR Collaboration], Phys. Rev. Lett. 94, 212302 (2005).

[6] J. Marton, M. Cargnelli, T. Ishiwatari, P. Kienle, K. Nikolic, E. Widmann, J. Zmeskal, M. Bazzi et al., IEEE Trans. Nucl. Sci. 56 (2009) 1400-1404.

[7] M. Bazzi et al., Nucl. Instrum. Meth. A 628, 264 (2011).

[8] T. Hyodo, W. Weise, D. Jido, L. Roca and A. Hosaka, Mod. Phys. Lett. A 23, 2393 (2008) [arXiv:0802.2212 [hep-ph]].

[9] W. Weise and R. Hartle, Nucl. Phys. A 804, 173 (2008) [arXiv:0801.1467 [nucl-th]].

[10] Y. Akaishi and T. Yamazaki, Phys. Rev. C65 (2002) 044005.

[11] T. Yamazaki et al., Phys. Rev. Lett. 104 (2010) 132502.

[12] A.N. Ivanov, P. Kienle, J. Marton, M. Pietschmann, http://arxiv.org/abs/1102.4163v1

[13] A. Filippi and S. Piano, Hyp. Interact. 193 (2009) 173.

[14] K. Suzuki et al., Hyp. Interact. 193 (2009) 189.

[15] T. Bressani, G. Bendiscioli, L. Lavezzi et al. Hyp. Interact. 193 (2009) 201.

[16] E. Oset et al., Phys.Rev. C74 (2006) 015207.

[17] O. Vazquez Doce et al., EPJ Web of Conferences 3, 03021 (2010)

[18] S. Ajimura et al., EPJ Web of Conferences 3, 07015 (2010)

[19] Mini-Proceedings, ECT* Workshop on Hadronic Atoms and Kaonic Nuclei, 2009, Eds. C. Curceanu and J. Marton, arXiv:1003.2328v2 [nucl-ex].

[20] http://www.hadronphysics2.eu/wp9.html

[21] http://www.hadronphysics2.eu

[22] http://www.lnf.infn.it/esperimenti/siddharta/

[23] M. Cargnelli et al., Nucl. Phys. A 835, 27 (2010).

[24] M. Cargnelli et al. [SIDDHARTA Collaboration] to be published.

[25] U. G. Meißner, U. Raha, A. Rusetsky, Eur. Phys. J. C35 (2004) 349-357. [hep-ph/0402261].

[26] V. Baru, E. Epelbaum and A. Rusetsky, Eur. Phys. J. A42 111, [arxiv:0905.4249 [nucl-th]] and [arxiv:0910.5385 [nucl-th]].

[27] M. Bazzi et al. [SIDDHARTA Collaboration] Phys. Lett. B681 (2009) 310.

[28] S. Okada et al., Phys. Lett B653 (2007) 387.

[29] M. Bazzi et al. [SIDDHARTA Collaboration], Phys. Lett. in print, http://arxiv.org/abs/1010.4631v2

[30] Y. Akaishi, Proc. EXA05 Conference, Vienna, 2005, eds. A. Hirtl, J. Marton, E.Widmann, J. Zmeskal, p.45.

[31] R.S. Hayano et at., Proposal of J-Parc 50 GeV PS (J-PARC E17) 2006. 
[32] M.P. Faifman, Mini-Proceedings, ECT* Workshop on Hadronic Atoms and Kaonic Nuclei, 2009, Eds. C. Curceanu and J. Marton, p. 11; arXiv:1003.2328v2 [nucl-ex].

[33] M. Faber, M. P. Faifman, A. N. Ivanov, J. Marton, M. Pitschmann, N. I. Troitskaya, [arXiv:1012.3933 [nucl-th]].

[34] R. C. Barrett, A. Deloff, Phys. Rev. C60 (1999) 025201.

[35] A. N. Ivanov, M. Cargnelli, M. Faber, H. Fuhrmann, V. A. Ivanova, J. Marton, N. I. Troitskaya, J. Zmeskal, Eur. Phys. J. A23 (2005) 79-111. [nucl-th/0406053].

[36] [SIDDHARTA2 Collaboration] SIDDHARTA2 Proposal, 2010.

[37] J. Siebenson [ HADES Collaboration ], AIP Conf. Proc. 1322 (2010) 389-393.

[38] K. Moriya [ CLAS Collaboration ], Prog. Theor. Phys. Suppl. 186 (2010) 234-239.

[39] S. Wycech, Int. J. Mod. Phys. A26 (2011) 402-407.

[40] K. Suzuki et al., Nucl.Phys.A827 (2009) 312C.

[41] N. Shevchenko et al. PRL 98 (2007) 082301.

[42] N. Shevchenko et al. PRC 76(2007) 044004.

[43] Y. Ikeda, T. Sato PRC 76 (2007) 035203.

[44] A. Dote, T. Hyodo, W. Weise NPA 804 (2008) 197.

[45] A. Dote, T. Hyodo, W. Weise PRC 79 (2009) 014003.

[46] O. Vázquez Doce, Hyperfine Interact. 193 (2009) 195.

[47] T. Tsukuda, EPJ Web of Conferences 3, 03016 (2010)

[48] http://www.hadronphysics2.eu/wp24.html

[49] http://www.hadronphysics2.eu/wp28.html 